\title{
Forced Rayleigh Scattering from Lipid-Water Smectic Phases
}

\section{Citation}

Chan, Winston, and Peter S. Pershan. 1977. Forced Rayleigh scattering from lipid-water smectic phases. Physical Review Letters 39(21): 1368-1371.

\section{Published Version}

doi:10.1103/PhysRevLett.39.1368

\section{Permanent link}

http://nrs.harvard.edu/urn-3:HUL.InstRepos:10361968

\section{Terms of Use}

This article was downloaded from Harvard University's DASH repository, and is made available under the terms and conditions applicable to Other Posted Material, as set forth at http:// nrs.harvard.edu/urn-3:HUL.InstRepos:dash.current.terms-of-use\#LAA

\section{Share Your Story}

The Harvard community has made this article openly available.

Please share how this access benefits you. Submit a story.

\section{Accessibility}


D. N. Langenberg, Phys. Rev. Lett. 33, 215 (1974).

${ }^{2}$ R. Janik, L. Morelli, N. C. Cirillo, Jr., J. N. Lechevet, and W. D. Gregory, IEEE Trans. Mag. 11, 687 (1975).

${ }^{3}$ J. Fuchs, P. W. Epperlein, M. Welte, and W. Eisenmenger, Phys. Rev. Lett. 38, 919 (1977).

${ }^{4}$ R. C. Dynes, V. Narayanamurti, and J. P. Garno, Phys. Rev. Lett. 39, 229 (1977).

${ }^{5}$ I. Iguchi, to be published.

${ }^{6}$ J. J. Chang and D. J. Scalapino, Phys. Rev. B 10 , 4047 (1974).

${ }^{7}$ L. N. Smith, J. Low Temp. Phys. 28, 519 (1977).

${ }^{8}$ C. S. Owen and D. J. Scalapino, Phys. Rev. Lett. 28 , 1559 (1972).
${ }^{9}$ B. A. Huberman, J. Chem. Phys. $\underline{65}, 2013$ (1976).

${ }^{10}$ A. I. Larkin and D. E. Khmel'nitskii, Zh. Eksp. Teor. Fiz. 55, 2345 (1968) [Sov. Phys. JETP 28, 1245 (1969)] .

${ }^{11}$ W. H. Parker, Phys. Rev. B 12 , 3667 (1975).

${ }^{12}$ The length scale of the instability, Eq. (11), actually varies as $A^{1 / 2} / B^{1 / 4}$. Thus, to the extent that $A$ and $B$ differ from unity, our result for this length will change. A microscopic theory is needed to determine $A$ and $B$, but we believe that for $0.3 \leqslant T / T_{c} \leqslant 0.9$ our estimate of unity sets the correct order of magnitude.

${ }^{13}$ A. Rothwarf and B. N. Taylor, Phys. Rev. Lett. 19, 27 (1967).

${ }^{14}$ If the density dependence of the driving currents is included, Eq. (8) becomes

$$
\left[\alpha+2 \tau_{k}^{-1}+D(q)-\partial \boldsymbol{I}_{\mathrm{qp}} / \partial N_{\mathrm{qp}}\right]\left(\alpha+\tau_{B}^{-1}+\tau_{\mathrm{es}}{ }^{-1}-\partial \boldsymbol{I}_{\mathrm{ph}} / \partial N_{\mathrm{ph}}\right)-\left(2 \tau_{K}^{-1}+\partial \boldsymbol{I}_{\mathrm{qp}} / \partial N_{\mathrm{qp}}\right)\left(\tau_{R}^{-1}-\partial \boldsymbol{I}_{\mathrm{ph}} / \partial N_{\mathrm{qp}}\right)=0 .
$$

${ }^{15}$ S. Chandrasekhar, Hydrodynamics and Hydrodynamic Stability (Clarendon, Oxford, 1961).

\title{
Forced Rayleigh Scattering from Lipid-Water Smectic Phases
}

\author{
Winston Chan ${ }^{(a)}$ and P. S. Pershan \\ Division of Applied Science, Gordon McKay Laboratory, Harvard University, Cambridge, Massachusetts 02138 \\ (Received 13 September 1977)
}

\begin{abstract}
The hydrodynamic relaxation rate for water inhomogeneities in aligned lipid-water smectics is measured for the first time using an improved version of the "forced Rayleigh" technique. Variation of the relaxation rate with water concentration yields a distinction between "bound" and "free" water.
\end{abstract}

Although part of the motivation for research into the physics of liquid crystals derives from the fact that many biological systems exhibit liquid crystalline morphology, physicists have by and large concerned themselves with studies of liquid crystalline systems that have little or no direct connection with biological systems. ${ }^{1,2}$ On the other hand, it is well known that lipid-water mixtures form smectic liquid crystal phases that have the same symmetries as liquid crystals that have been extensively studied. ${ }^{3,4}$ The lipid molecule possesses a hydrophilic polar head group that is attached to one or more long hydrophobic hydrocarbon chains. ${ }^{5}$ In the presence of water these amphiphilic lipid molecules can form planar bilayers in which the polar head groups locate on the surface so as to shield the hydrophobic chains from the water. Lipids are principal ingredients in biological membranes and lipid bilayers have been extensively studied as model membrane systems. Furthermore, studies on living systems have presented convincing evidence that many of the functions of living membranes are altered at temperatures that correspond to phase transitions in the smectic lipid-water (SLW) systems. ${ }^{5}$ Interactions between lipid and water are essential to the structure and stability of membranes. ${ }^{6}$ We report here new measurements on the fluidity of water in the SLW phase of dipalmitoyl phosphatidylcholine (DPPC).

SLW systems consist of a stack of planar lipid bilayers separated from each other by a layer of water. ${ }^{7}$ Tecniques have recently been developed to produce macroscopic samples $\sim 1 \mathrm{~cm}^{2}$ in area and $\sim 0.1 \mu \mathrm{m}$ thick. ${ }^{8,9}$ The size and quality of these samples are comparable to other liquid crystals and crystals that have been studied by physical techniques. These systems also have interesting physical properties without regard to their biological relevance. For example, a unifed hydrodynamic theory of crystals, liquid crystals, and liquids has been developed and experiments have been done to observe some of the modes that have been predicted for single-component, nonbiological liquid crystals. ${ }^{1,2,10}$ de Gennes and Brochard have applied these general ideas to the specific case of the binary lipid-water smectic and made predictions concerning an overdamped 
hydrodynamic mode that corresponds to the relaxation of fluctuations in the local water concentration. ${ }^{11}$ We present here the results of the first experimental observation of that mode.

The experimental technique is a new, more powerful variation on the forced Rayleigh technique (FRT). ${ }^{12-14}$ The present experiment would not have been possible without this improvement. In the conventional FRT two beams from the same laser (in our case an argon ion laser, $\lambda=5145 \AA$ ), making an angle $\theta$ with respect to each other, and incident on one spot in the sample form an interference pattern with intensity distribution

$$
I=F(x, y, z)\left\{1+\cos \left[q_{0} x+\psi\right]\right\},
$$

where $q_{0}=4 \pi \lambda^{-1} \sin (\theta / 2)$ and $\lambda$ is the laser wavelength. The function $F(x, y, z)$ describes the beam width and average intensity distribution. The phase factor $\psi$ determines where the maxima and minima in the interference pattern are located. A change in path length between laser and sample for one beam relative to the other by $\lambda / 2$ will change $\psi$ by $\pi$. If the sample absorbs power from the incident beam, local heating will give rise to a variation in the local index of refraction that will induce a phase grating in the material. In the conventional FRT the heating laser is blocked and one observes the decay in intensity of a Bragg diffraction pattern of the light from a second laser (He-Ne in our case).

The principal limitation on conventional FRT is that in addition to Bragg diffraction from the grating induced by the periodic part of $I$ [Eq. (1)] there is also non-Bragg diffraction from the inhomogeneity induced by the more slowly varying function $F(x, y, z$,$) . For wave vector \vec{q}$ the spatial Fourier transformation of the index inhomogeneity $\delta(q)$ is proportional to the temperature $\delta T(q)$, and thermal conductivity yields $\delta T(q) \sim q^{-2} I(q)$. Since the beam width $d$ is much larger than the fringe separation $2 \pi q_{0}{ }^{-1}$, the low spatial frequencies with $q \sim 2 \pi d^{-1}$ have $\delta T(q) \gg \delta T\left(q_{0}\right)$. For the two-component lipid-water systems the FRT would detect slow water relaxation $\tau_{w}(q)^{-1}=D q^{2}$ and slow thermal relaxation $\tau_{T}(q)^{-1}=\chi q^{2}$ as well as two fast relaxations (at $q_{0} \gg q$ ) of the Bragg diffraction due to both thermal and water decays. ${ }^{10,11}$ As we will show below, $\chi \gg D$ and the time constant of the slow thermal decay is comparable to the time constant of the fast water decay. In addition the signal due to the slow thermal decay is some orders of magnitude larger than the signal due to the fast water decay.

To overcome this problem we detect the decay- ing signal using an optical heterodyne scheme. ${ }^{14}$ The phase of the electromagnetic wave diffracted by the periodic term is sensitive to the phase $\psi$ in Eq. (1). Varying $\psi$ from 0 to $\pi$ changes the sign of the Bragg term in the heterodyne detection scheme without changing the sign of the low$q$ terms. The measurement is done repeatedly, with $\psi$ alternately set at 0 or $\pi$, and the decay signals are added to the memory of a digital system when $\psi=0$ and subtracted from the memory when $\psi=\pi$. Signals arising from the first term in Eq. (1) average to zero and the only remaining signal is due to the Bragg diffraction from the periodic term. Figure 1 shows the result of this process for a thermal decay signal and for a water decay. The scatter in data points is a measure of the shot noise due to the reference beam. Typically Bragg signals whose intensitites would be less than the background signal by $10^{-3}$ if homodyne detection was used are easily detected with the present technique.

In order to demonstrate the observed relaxation rates satisfy the $q^{2}$ law the experiment was done as a function of $\theta$, i.e., as a function of $q_{0}$ with $\vec{q}_{0}$ always in the bilayer planes. Figure 2 shows typical results for a sample containing $24 \%$ water by weight at $T=70^{\circ} \mathrm{C}$. The decay rates are linear in $q_{0}{ }^{2}$, except for $q_{0} \lesssim 2 \pi$ (sample thickness) ${ }^{-1}$ where temperature relaxation occurs through heat flow into the glass substrates. Figure 3 illustrates the temperature dependence of $D$ for DPPC samples containing varying weight percentage of wa-

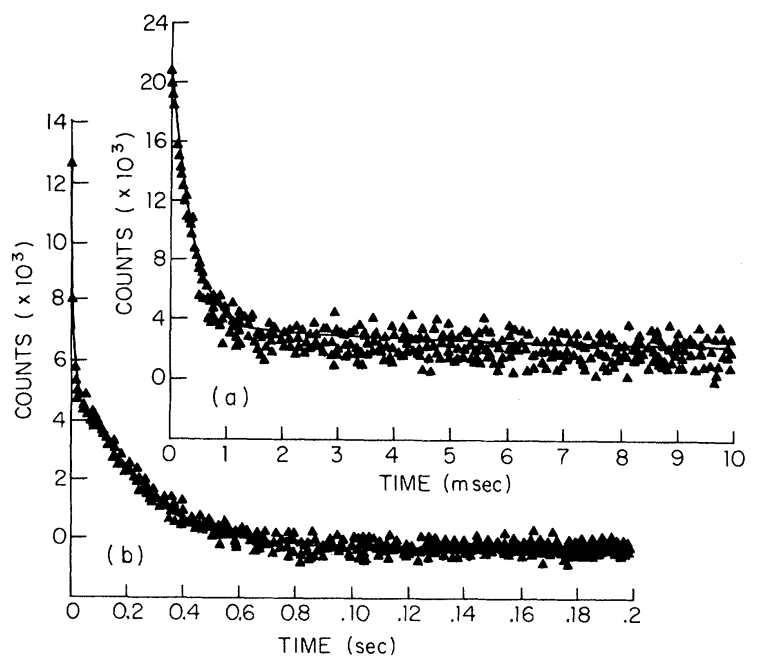

FIG. 1. Typical example of forced Rayleigh signals for (a) thermal diffusivity and (b) water concentration decay. The sample was at $70^{\circ} \mathrm{C}$ and contained $21 \%$ water by weight. 


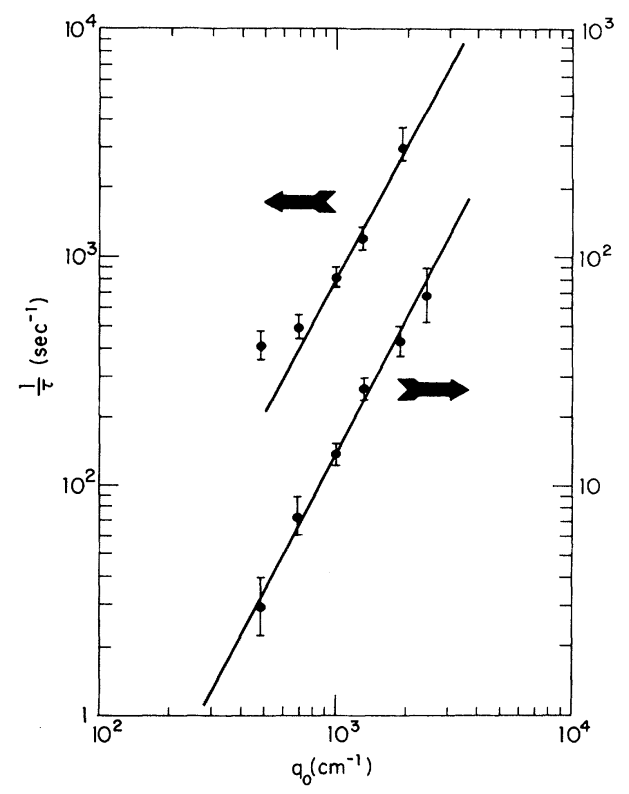

FIG. 2. Typical plots of observed decay rates vs $q_{0}$ for a sample at $70^{\circ} \mathrm{C}$ containing $24 \%$ water by weight. The upper data correspond to thermal diffusivity and the lower to water decay. The solid lines indicate $q^{2}$ dependence. Sample thickness was $\sim 125 \mu \mathrm{m}$.

ter. Except for the two lowest temperature points all the data were taken in the $L \alpha$ or smectic- $A$ phase of DPPC. ${ }^{3,4,7,9}$

It is generally agreed that at low concentrations the water is bound to the polar head group of the lipid and the chemical potential of the water in the lipid is significantly lower than that of bulk water at the same temperature and pressure. , $4,7,15-21^{-1}$ With increasing concentration the additional water is less strongly bound and the chemical potential of the water increases. The question of interest is whether the physical properties of the water in the lipid are ever the same as for bulk water. The data in Fig. 3 give a partial answer to this.

The relative strength of the concentration relaxation mode to the thermal mode is considerably greater in the SLW system than it would be in an isotropic liquid because the thickness of the lipid bilayer decreases with increasing temperature. ${ }^{3,4,7}$ Assuming that the sum of the water layer thickness and the lipid bilayer thickness remain fixed, nonuniform heating induces nonuniform thickness for the lipid bilayer with water flowing from regions of lower to higher local temperature. The index of refraction of bulk water is less than that of pure hydrocarbon so that the water flow causes the index of refraction in the hotter region to de-

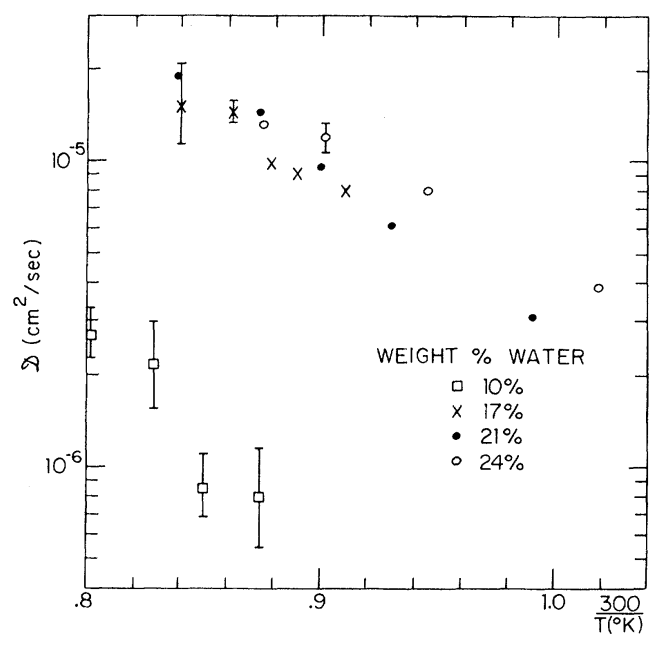

FIG. 3. Temperature dependence of $D$ for samples containing varying amounts of water. Error bars are indicated for representative points.

crease relative to the cooler. Even without water flow the hotter region should have a lower index of refraction than the cooler because normal thermal expansion would make the hotter region less dense. Consistent with this we observe that at high water content the thermal decay and the water decay signals have the same relative sign. For lower water content (less than 10\%) this is not always true.

Brochard and de Gennes ${ }^{11}$ developed a theoretical prediction for the value of $D$ based on the assumption that elastic pressure caused by the deformed lipid bilayer forces water to flow. Unfortunately this model involves elastic parameters that are not known. Starting from the general hydrodynamic formalism ${ }^{10}$ and without making any assumptions about water flow one can obtain

$$
D=\delta^{-1}(n * \partial \mu / \partial n *),
$$

where $\delta$ is a phenomenological coefficient, $n *$ is the number of water molecules per unit area in the lipid, and $\mu$ is the chemical potential for water in the lipid. Elworthy has measured $\mu$ versus water concentration for both egg lecithin and DPPC. At $40^{\circ} \mathrm{C}$ and $20 \%$ water, he obtains $n * \partial \mu$ / $\partial n * \sim 9 \times 10^{-15}$ erg with little temperature dependence for either lipid. ${ }^{15}$ Using a different technique LeNeveau et al., obtained values of similar magnitude for egg lecithin. ${ }^{16}$ The coefficient $\delta$ can be obtained from the data in Fig. 3 and these chemical potentials. The assumptions about the water flow made by Brochard and de Gennes yield $\delta^{-1}=(12 \eta v)^{-1} d_{w}{ }^{2}$ where $\eta$ is the viscosity of 
water, $v$ is its molecular volume, and $d_{w}$ is the water thickness. Taking $\eta=0.4 \mathrm{cp}$ and $v=3 \times 10^{-23}$ $\mathrm{cm}^{3}$, the data in Fig. 3 for $21 \%$ water and $70^{\circ} \mathrm{C}$ yield $d_{w}=4.4 \AA$ using Elworthy's results and $d_{w}$ $=1.7 \AA$ using Rand's.

Small's analysis of x-ray diffraction results for egg lecithin gives a value of $23.8 \AA$ for the full thickness of a water layer at $20 \%$ water; however, since a fraction of the water is "bound to the lipid" he argued that only $1.8 \AA$ of this should correspond to "free water." The agreement with our result supports his interpretation. For lower water concentrations the above analysis of our data indicates $d_{w} \sim 0$ at $10 \%$ water. This is consistent with theoretical models for the number of water molecules required to fully hydrate the DPPC polar head group. ${ }^{7}$

The values of $D$ shown in Fig. 3 can be fitted to an Arrhenius law with activation energies of 0.25 to $0.45 \mathrm{eV}$, decreasing with increasing $n *$. This can be compared to the activation energy for the viscosity of water, $0.17 \mathrm{eV}$. Both the absolute values and the trend with increasing $n *$ are consistent with some degree of binding between water and the polar head group.

With the deductions that (1) $d_{w}$ is considerably smaller than the approximately $20 \AA$ that may be considered the water layer and (2) the activation energy is twice that in bulk water, we conclude that much of the water is relatively bound and is significantly less mobile than free water.

We would like to express our appreciation to Dr. E. A. Dawidowicz of the Harvard Medical School for instruction in methods of purifying our lipid materials. We also would like to express our appreciation to Dr. Sanford Asher for help in other chemical aspects of this project. Dr. Linda Powers was of invaluable help in providing instructions on the techniques of making aligned samples. This work has been supported in part by the Joint Services Electronics Program (U.S. Army, Navy, and Air Force) under Contract No. N00014-75-C-0648 and by the National Science
Foundation under Grants No. DMR-76-01111 and No. DMR-76-22452.

${ }^{(a)}$ Present address: Clark Hall, Cornell University,
Ithaca, N.Y. 14850 .
${ }^{1}$ P. G. de Gennes, The Physics of Liquid Crystals
(Clarendon Press, Oxford, 1974).
${ }^{2}$ H. Gasparoux and J. Prost, Annu. Rev. Phys. Chem. 27, 175 (1976) .

${ }^{3}$ D. Chapman, Quart. Rev. Biophys. 8,185 (1975).

${ }^{4}$ V. Luzzati, A. Tardieu, Annu. Rev. Phys. Chem. 25, 79 (1974).

${ }^{5}$ Albert L. Lehninger, Biochemistry (Worth, New York, 1975), Chap. 11.

${ }^{6}$ S. J. Singer, in Structure and Function of Biological Membranes, edited by L. I. Rothfield (Academic, New York, 1971).

${ }^{7}$ D. M. Small, J. Lipid Res. $\underline{8}, 551$ (1967).

${ }^{8}$ L. Powers and N. A. Clark, Proc. Nat. Acad. Sci. U.S.A. 72, 840 (1975).

${ }^{9}$ L. Powers and P. S. Pershan, to be published.

${ }^{10}$ P. C. Martin, O. Parodi, and P. S. Pershan, Phys. Rev. A $\underline{6}, 2401$ (1972).

${ }^{11}$ F. Brochard, and P. G. de Gennes, Pramana, Suppl. No. 1,1 (1975).

${ }^{12} \mathrm{~J}$. A. Cowen, C. Allain, and P. Lallemand, J. Phys. (Paris), Lett. 37, 313 (1976).

${ }^{13}$ H. Eichler, G. Salje, and H. Stahl, J. App. Phys. 44, 5383 (1973).

${ }^{14}$ D. W. Pohl, S. E. Schwarz, and V. Irniger, Phys. Rev. Lett. 31, 32 (1973); D. W. Pohl and V. Irniger, Phys. Rev. Lett. 36, 480 (1976).

${ }^{15}$ P. H. Elworthy, J. Chem. Soc. 1961, 5385, and $1962,4897$.

${ }^{16}$ D. M. LeNeveau, R. P. Rand, V. A. Parsegian, and D. Gingell, Biophys. J. 18, 209 (1977).

${ }^{17}$ Y. Lange and C. M. Gary Bobo, J. Gen. Physiol. $\underline{63}$, 690 (1974).

${ }^{18}$ R. G. Griffin, J. Am. Chem. Soc. 98, 851 (1976).

${ }^{19}$ A. Sanson, M. Ptak, J. L. Rigaud, and C. M. Gary

Bobo, Chem. Phys. Lipids 17, 445 (1976).

${ }^{20}$ E. G. Finer and A. Darke, Chem. Phys. Lipids $\underline{12}$, 1 (1974).

${ }^{21}$ Paul T. Inglefield, Kenneth A. Lindblom, and Albert M. Gottlieb, Biochem. Biophys. Acta 419, 196 (1976). 